\title{
Coronary Artery Calcification Progression Is Associated with Arterial Stiffness and Cardiac Repolarization Deterioration in Hemodialysis Patients
}

\author{
Biagio Di lorio ${ }^{a} \quad$ Onorio Nargi $^{b} \quad$ Emanuele Cucciniello ${ }^{a}$ Vincenzo Bellizzi $^{a}$ \\ Serena Torraca ${ }^{a}$ Domenico Russo $^{c}$ Antonio Bellasid ${ }^{d}$ \\ on behalf of the INDEPENDENT study investigators \\ a UO di Nefrologia e bUO di Diagnostica per Immagini, PO 'A Landolfi', Solofra, 'Cattedra di Nefrologia, \\ Università Federico II, Napoli, e dUOC di Nefrologia, Dialisi ed Ipertensione, Policlinico S. Orsola-Malpighi, \\ Azienda Ospedaliero-Universitaria, Bologna, Italia
}

\section{Key Words}

Coronary artery calcification - Progressor $\cdot$ QT dispersion

\begin{abstract}
Background/Aims: Evidence suggests that vascular calcification (VC) portends poor cardiovascular (CV) prognosis in patients undergoing maintenance dialysis (CKD-5). Nonetheless, how VC might predispose to CV mortality still remains to be clarified. Herein, we report on the association between coronary artery calcification (CAC) progression and changes in cardiac repolarization as well as arterial stiffness. Methods: 132 patients new to dialysis were identified. Demographic and clinical characteristics were collected at study entry and during the 12-month follow-up. CAC, 12lead ECG and pulse wave velocity (PWV) were assessed at baseline and study completion. Uni- and multivariable analyses were applied to detect factors associated with worsening of cardiac repolarization (QTd) and arterial stiffness (PWV). Results: Uni- and multivariable analyses revealed that CAC progression was associated with a significant increase in both QTd and PWV. Every 20-unit increase in the CAC score corresponded to a significant $23 \%(95 \% \mathrm{Cl} 1.12-$ $1.27 ; \mathrm{p}<0.001)$ and $32 \%(95 \% \mathrm{Cl} 1.09-1.37 ; \mathrm{p}<0.01)$ increase in the risk of experiencing a $1-\mathrm{m} / \mathrm{s}$ increase in PWV and $1 \mathrm{~ms}$
\end{abstract}

in QTd, respectively. Conclusion: VC is a marker of vasculopathy and appears to be associated with cardiac repolarization and arterial stiffness abnormalities in CKD- 5 patients.

Copyright $\odot 2011$ S. Karger AG, Basel

\section{Introduction}

Despite the fact that traditional cardiovascular (CV) risk factors are significantly over-represented in patients with chronic kidney disease (CKD) on maintenance dialysis (CKD-5), they do not seem to fully explain the incidence of cardiovascular diseases (CVD) observed in CKD-5 [1]. Hence, over the past decade a plethora of non-

INDEPENDENT study investigators: Filippo Aucella (S. Giovanni Rotondo), Pasquale Guastaferro and Angela di Gianni (S. Angelo dè Lombardi), Luigi Chiuchiulo (Avellino, Dialysis), Vincenzo Tedesco (Montella), Mario Migliorati (S. Giorgio A Cremano), Walter de Simone and Bruno Zito (Avellino), Ernesto d'Avanzo, Sara Bortone, Paola Nargi and Francesco Saverio lannaccone (Solofra), Patrizia Veniero, Maria Capuano and Raffaele Genualdo (Napoli: Pellegrini), Magda Lorenzo, Domenico Santoro, Ferdinando Avella (Nola), Luigi Morrone and Vinicio Martignetti (Benevento), Carmine Piscopo (Solofra), Domenico Matarese and Domenico Vigilante (Lucera), Assunta Aquino, Rosa Martino, Struzziero Giuseppe, Alfonso Frallicciardi, and Raffaele Tortoriello.

\section{KARGER \\ Fax +41613061234 E-Mail karger@karger.ch} www.karger.com

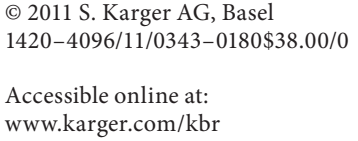

\author{
Biagio Di Iorio \\ UO di Nefrologia, PO 'A Landolfi' \\ Via Melito, snc \\ IT-83029 Solofra (Italy) \\ E-Mail br.diiorio@gmail.com
}


traditional risk factors and markers of vasculopathy have been indentified in the hope of providing nephrologists with new insights into the pathophysiology of CVD in CKD-5 and, possibly, with the therapeutic target of improving CV survival [1]. In this regard, several lines of evidence have repeatedly suggested that abnormalities in mineral metabolism (i.e. serum levels of calcium, phosphate, parathyroid hormone and vitamin $\mathrm{D}$ ), vascular calcification (VC) and poor CV outcome might be associated [2-4]. Hence, a new syndrome called chronic kidney disease mineral bone disorders was defined [5].

To date, several aspects of the link between mineral metabolism derangements, VC and mortality remain to be elucidated. In vitro experiments provide convincing evidence that calcium and phosphorous can trigger VC deposition and progression [6-9]. Several cross-sectional studies corroborate the notion that abnormalities in the serum levels of mineral metabolism parameters are associated with both VC and reduced survival [10-13]. In the light of these data, VC may represent a plausible explanation for the association between bone mineral imbalances and mortality. However, the debate on how VC might predispose to CV mortality is still open.

In a previous paper we reported on an independent association between coronary artery calcification (CAC) and electrocardiogram (ECG) abnormalities (i.e. QT dispersion (QTd)), suggesting that VC may affect cardiac repolarization and may predispose to cardiac arrhythmia [14-16]. However, to the best of our knowledge, no study has ever looked into these associations prospectively. Herein, we present the results of the impact of CAC progression on QTd and arterial stiffness of large arteries estimated by aortic pulse wave velocity (PWV).

\section{Materials and Methods}

Data presented in this paper have been extrapolated from the ongoing INDEPENDENT study database [17]. Briefly, the INDEPENDENT study is a randomized controlled trial designed to investigate the impact of two different phosphate binder regimes (sevelamer $\mathrm{HCl}$ vs. calcium or aluminum salts) on QT interval dispersion and CAC progression. Herein, we present a post hoc analysis aimed at testing in a longitudinal cohort the hypothesis that CAC progression is linked to an increase in arterial stiffness and cardiac repolarization abnormalities.

Inclusion criteria were a history of CKD requiring dialysis for $<120$ days. Exclusion criteria included age $>75$ years, concomitant acute illness or history of cardiac arrhythmia, history of coronary artery bypass, liver dysfunction and hypothyroidism and use of drugs that prolong the QT interval. Enrollment began in September 2007 and continued through July 2009. Written informed consent was obtained from all participants before study-related proce- dures were performed and after approval from each institutional ethical review board. All the procedures involved in the study were conducted in compliance with the Declaration of Helsinki, Ethical Principles for Medical Research Involving Human Subjects.

The investigators were instructed to control blood pressure ( $\leq 130 / 80 \mathrm{~mm} \mathrm{Hg})$, anemia $(\mathrm{Hb}>11 \mathrm{~g} / \mathrm{dl}$, TSAT $>20 \%)$, acidosis $\left(\mathrm{HCO}_{3}\right.$ between 20 and $\left.24 \mathrm{mmol} / \mathrm{l}\right)$, diabetes (HbAlc $\left.<7.0 \%\right)$, dyslipidemia (total cholesterol $<200 \mathrm{mg} / \mathrm{dl}$; LDL cholesterol $<100$ $\mathrm{mg} / \mathrm{dl}$; triglycerides $<180 \mathrm{mg} / \mathrm{dl}$ ), and the parameters of bone mineral metabolism (serum phosphorous, calcium, and iPTH between $2.5-5.0,8.0-9.9$, and $150-300 \mathrm{pg} / \mathrm{ml}$, respectively) according to current guidelines.

Routine biochemical laboratory measurements were obtained at baseline and at 6-monthly intervals. All blood samples were taken before the midweek dialysis session and after $12 \mathrm{~h}$ fasting. Serum parameters of mineral metabolism, electrolytes, inflammation, anemia, liver function and dialysis adequacy were performed by the usual laboratories of the facilities.

CAC score (CACS), PWV and QTd were evaluated at study entry and after 12 months. CACS was assessed by a multislice lightspeed (GE Medical Systems) equipment at one center (Solofra, Italy). A standard imaging protocol was used to acquire a set of ECG-gated tomographic slices from the carina to the diaphragm. A CACS was calculated via the Agatston score as previously described. Two independent readers scored all scans.

QTd was assessed on a 12-channel recorder (Esaote Biomedicals) at a paper speed and gain of $25 \mathrm{~mm} / \mathrm{s}$ and $10 \mathrm{~mm} / \mathrm{mV}$, respectively. To minimize the QT assessment variability, all QT assessments were performed at the same time of the day and read by one single experienced investigator who was blinded to other clinical data. QT was measured as the distance between the beginning of the QRS complex and the end of the T wave. Moreover, each QT interval was also corrected for the heart rate using the Bazett's formula $(\mathrm{QTC}=\mathrm{QT} / \mathrm{RR})$. The difference between the maximum QTc and the minimum QTc was also calculated and represents the corrected QT interval dispersion (QTcd).

Carotid-femoral PWV was evaluated by applanation tonometry with Pulse Pen (Diatecne, Milan, Italy). Briefly, PWV is the velocity at which the sphygmic wave generated at each heart beat travels along the arterial tree. It is estimated by dividing the distance between the heart and the recording sites by the time interval between the cardiac systole and the pulse upstroke at the recording site (carotid and femoral artery). An estimate of the distance travelled is obtained by measuring the distance on the body surface between the sternal notch and the carotid artery and between the sternal notch and the groin. With the use of a tonometer, the time between the systole and the carotid and femoral upstroke is measured as the time interval between the $\mathrm{R}$ wave on the ECG and the upstroke at the carotid and femoral artery.

\section{Statistical Methods}

Demographic, clinical and laboratory characteristics were collected at enrollment and compared between patients with and without progression of CACS after a 12-month follow-up. Atherosclerotic disease (ASCVD) was defined if any of the following clinical data was reported in the patient's chart: history of cerebrovascular disease; peripheral vascular disease; angina pectoris; history of myocardial infarction; aortic aneurysm; history of percutaneous coronary angioplasty with or without stenting. Owing to the skewness of the CAC variable, CAC progression was defined de- 
Table 1. Demographic and clinical characteristic of the study cohort according to CAC progression status at baseline

\begin{tabular}{|c|c|c|c|c|}
\hline & $\begin{array}{l}\text { All } \\
\text { population } \\
(\mathrm{n}=132)\end{array}$ & $\begin{array}{l}\text { Progressor } \\
(\mathrm{n}=81)\end{array}$ & $\begin{array}{l}\text { Non- } \\
\text { progressor } \\
(\mathrm{n}=51)\end{array}$ & $\begin{array}{l}\mathrm{p} \\
\text { value }\end{array}$ \\
\hline Males, \% & 64 & 61 & 72 & 0.259 \\
\hline Age, years & $65.3 \pm 16.6$ & $66.8 \pm 16.8$ & $63.2 \pm 16.5$ & 0.23 \\
\hline ASCVD, \% & 34 & 42 & 23 & 0.041 \\
\hline Hypertension, \% & 79.5 & 84.8 & 71.7 & 0.109 \\
\hline Diabetes, \% & 24 & 30 & 13 & 0.042 \\
\hline $\mathrm{SBP}, \mathrm{mm} \mathrm{Hg}$ & $135 \pm 16$ & $130 \pm 34$ & $134 \pm 16$ & 0.433 \\
\hline $\mathrm{DBP}, \mathrm{mm} \mathrm{Hg}$ & $75 \pm 10$ & $74 \pm 10$ & $74 \pm 11$ & 1 \\
\hline Phosphorous, mg/dl & $4.9 \pm 1.5$ & $4.8 \pm 1.4$ & $5.0 \pm 1.5$ & 0.438 \\
\hline Calcium, mg/dl & $8.7 \pm 1.1$ & $8.7 \pm 1.0$ & $8.6 \pm 1.1$ & 0.591 \\
\hline Hemoglobin, g/dl & $10.9 \pm 1.9$ & $11.0 \pm 1.8$ & $10.7 \pm 2.1$ & 0.383 \\
\hline Intact PTH, pg/mmol & $335 \pm 245$ & $326 \pm 234$ & $342 \pm 264$ & 0.717 \\
\hline Albumin, $\mathrm{g} / \mathrm{dl}$ & $3.7 \pm 0.5$ & $3.7 \pm 0.6$ & $3.7 \pm 0.5$ & 1 \\
\hline LDL cholesterol, mg/dl & $101 \pm 33$ & $104 \pm 26$ & $97 \pm 39$ & 0.218 \\
\hline $\mathrm{CRP}, \mathrm{mg} / \mathrm{dl}$ & $8.3 \pm 11.3$ & $10.5 \pm 11.5$ & $6.8 \pm 7.6$ & 0.044 \\
\hline CACS & $286 \pm 744$ & $808 \pm 1,221$ & $151 \pm 273$ & 0.00001 \\
\hline $\mathrm{PWV}, \mathrm{m} / \mathrm{s}$ & $8.2 \pm 1.9$ & $8.8 \pm 1.7$ & $7.5 \pm 1.9$ & 0.00001 \\
\hline QTc, ms & $406 \pm 36$ & $411 \pm 25$ & $400 \pm 47$ & 0.082 \\
\hline QTd, ms & $23 \pm 9$ & $23 \pm 9$ & $24 \pm 9$ & 0.535 \\
\hline QTd $>60 \mathrm{~ms}$ & $24 \pm 9$ & $24 \pm 10$ & $25 \pm 11$ & 0.591 \\
\hline
\end{tabular}

ASCVD = Cerebrovascular disease; peripheral vascular disease; angina pectoris; history of myocardial infarction; aortic aneurysm; history of percutaneous coronary angioplasty with or without stenting; SBP = systolic blood pressure; DBP = diastolic blood pressure; $\mathrm{CRP}=\mathrm{C}$-reactive protein; $\mathrm{CAC}=$ coronary artery calcium score; $\mathrm{LDL}=$ low-density lipoprotein cholesterol; $\mathrm{PWV}=$ pulse wave velocity; QTc = corrected QT interval; QTd = QT dispersion.

pending on the baseline CACS as previously described [18]. Briefly, for patients with CACS $<30$ at baseline, true progression was considered present if CACS was $\geq 30$ at study completion. For patients with a baseline CACS $>30$, a relative increase from baseline $\geq 15 \%$ /year was considered evidence of true progression as described in previous publications. In the text, patients with and without signs of true progression were defined as progressors and non-progressors, respectively. Contrariwise, PWV and QTd change are expressed as a relative change from the baseline.

Data are expressed as mean \pm SD unless otherwise specified; $t$ test and other non-parametric tests were used to compare the two treatment groups when appropriate. Spearman's correlation coefficient was used to assess a linear association between changes in CAC and other markers of CVD (i.e. PWV and QTd). All significant variables at univariable analyses were included in the multivariable models. Logistic regression was used to detect factors independently associated with CAC progression (age, history of hypertension, diabetes, ASCVD, C-reactive protein (CRP), LDL cholesterol and sevelamer use were entered in the multivariable model). In addition, linear regression was used to determine factors associated with QT and PWV change (i.e. age, history of hypertension, diabetes, ASCVD, CRP, LDL cholesterol, sevelamer use and $C A C$ progression were entered in the multivariable model). A p value $<0.05$ was considered statistically significant. All analyses were performed with SAS 9.0.

\section{Results}

From the original study cohort of the INDEPENDENT study, we identified all subjects who had completed the study follow-up at the time of the analyses were completed. Notably, there were not significant differences between those patients who did and did not complete the study follow-up (data not shown). A total of 152 patients were identified for this study. 16 (10.5\%) patients expired and $4(2.6 \%)$ other patients were excluded because of a pacemaker implantation before study completion.

Demographic, clinical and laboratory characteristics of the 132 patients who completed the study follow-up are summarized in tables 1 and 2. Overall, the study cohort consisted of middle-aged (65.3 \pm 16.6 years) men and women (64 and $36 \%$ of the entire study cohort, respectively). As expected, diabetes ( $23 \%$ of the entire study cohort), arterial hypertension (79.5\%), and ASCVD (34\%) were the most common comorbidities recorded.

At dialysis entry, 93 (70.4\%) patients showed evidence of CAC. Specifically, 35 (26.5\%), 14 (10.6\%) and 44 (33.3\%) 
Table 2. Demographic and clinical characteristic of the study cohort at baseline and at study completion

\begin{tabular}{lcccccc}
\hline & \multicolumn{2}{l}{ Progressor $(\mathrm{n}=81)$} & & \multicolumn{2}{c}{ Non-progressor $(\mathrm{n}=51)$} & \multirow{2}{*}{ p value } \\
\cline { 2 - 3 } & baseline & follow-up & & baseline & follow-up & \\
\hline SBP, mm Hg & $130 \pm 34$ & $135 \pm 20$ & & $134 \pm 16$ & $132 \pm 25$ & 0.448 \\
DBP, mm Hg & $74 \pm 10$ & $75 \pm 10$ & & $74 \pm 11$ & $72 \pm 11$ & 0.109 \\
Phosphorous, mg/dl & $4.8 \pm 1.4$ & $4.9 \pm 1.5$ & & $5.0 \pm 1.5$ & $4.2 \pm 1.3^{*}$ & 0.007 \\
Calcium, mg/dl & $8.7 \pm 1.0$ & $8.9 \pm 1.0$ & & $8.6 \pm 1.1$ & $9.2 \pm 0.9^{*}$ & 0.084 \\
Hemoglobin, g/dl & $11.0 \pm 1.8$ & $11.4 \pm 1.3$ & & $10.7 \pm 2.1$ & $12.2 \pm 1.5^{*}$ & 0.002 \\
Intact PTH, pg/mmol & $326 \pm 234$ & $377 \pm 244$ & & $342 \pm 264$ & $294 \pm 200^{*}$ & 0.044 \\
Albumin, g/dl & $3.7 \pm 0.6$ & $3.7 \pm 0.5$ & & $3.7 \pm 0.5$ & $3.9 \pm 0.5^{*}$ & 0.027 \\
LDL cholesterol, mg/dl & $104 \pm 26$ & $106 \pm 27$ & & $97 \pm 39$ & $98 \pm 24$ & 0.086 \\
CRP, mg/dl & $10.5 \pm 11.5$ & $11.5 \pm 9.3$ & & $6.8 \pm 7.6$ & $6.9 \pm 5.8$ & 0.002 \\
CACS & $808 \pm 1,221$ & $964 \pm 1,322$ & & $151 \pm 273$ & $159 \pm 267$ & 0.0001 \\
PWV, m/s & $8.8 \pm 1.7$ & $11.0 \pm 2.7^{*}$ & & $7.5 \pm 1.9$ & $8.7 \pm 2.0$ & 0.0001 \\
QTc, ms & $411 \pm 25$ & $420 \pm 35$ & & $400 \pm 47$ & $393 \pm 38$ & 0.0001 \\
QTd, ms & $23 \pm 9$ & $33 \pm 20^{*}$ & & $24 \pm 9$ & $21 \pm 11$ & 0.0001 \\
QTd $>60 \mathrm{~ms}$ & $12.3 \%$ & $48.1 \%$ & & $9.8 \%$ & $13.7 \%$ & 0.001 \\
\hline
\end{tabular}

$\mathrm{p}$ values refer to between-group comparisons at study completion.

${ }^{*} \mathrm{p}<0.05$ within-group change at study completion. See table 1 for abbreviations.

exhibited an Agatston CACS of 0-100, 100-400 and $>400$, respectively (fig. 1). True progression of CAC was detected in $81(61 \%)$ patients. As reported in table 1, progressors were on average older and more likely to be diabetic. They had a greater burden of coronary artery disease and higher levels of serum CRP at baseline. Instead, at study completion, progressors exhibited lower levels of serum albumin and a less favorable mineral metabolism control (table2). A significantlygreaterproportion of non-progressors were treated with sevelamer $\mathrm{HCl}$ when compared to progressors ( 73 vs. $18 \%, p<0.001)$. During the study followup there was no difference in blood pressure, anemia, acidosis, diabetes lipid and mineral metabolism control between progressors and non-progressors (data not shown).

After adjustment for risk factors, CAC progression was independently associated with diabetes (odds ratio (OR) diabetes vs. non-diabetes 1.17; 95\% CI 1.13-1.28; $\mathrm{p}=0.01$ ), serum LDL cholesterol (OR per 10-mg/dl increase 1.07; 95\% CI 1.01-1.12; $\mathrm{p}=0.05)$, serum CRP (OR per 5-mg/dl increase 1.09; 95\% CI 1.04-1.18; $\mathrm{p}=0.05)$, use of sevelamer $\mathrm{HCl}$ (OR 0.66; 95\% CI 0.36-0.81; p < 0.001) (table 3).

As reported in table 2, a significant change in CAC burden was associated with changes in arterial stiffness and cardiac repolarization. Despite the fact that there was no difference in PWV and QTcd between groups at baseline, CAC progression was associated with a significantly greater increase in PWV and QTd at study completion (table 2). A significant increase in arterial stiffness was

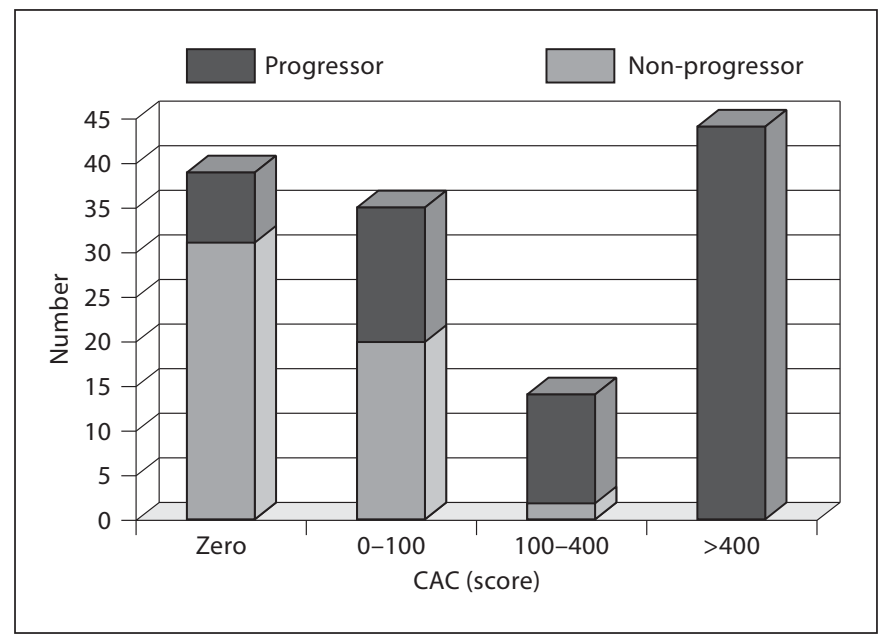

Fig. 1. CAC burden at dialysis entry and its association with CAC progression by study completion.

noted among progressors but not among non-progressors (PWV increased from $8.8 \pm 1.7$ to $11.0 \pm 2.7 \mathrm{~m} / \mathrm{s}$, $\mathrm{p}<0.05$, and from $7.5 \pm 1.9$ to $8.7 \pm 2.0 \mathrm{~m} / \mathrm{s}, \mathrm{p}=\mathrm{NS}$, in progressors and non-progressors, respectively). Similarly, at study completion, QTd was increased among progressors but not among non-progressors (QTd increased from $23 \pm 9$ to $33 \pm 20 \mathrm{~ms}, \mathrm{p}<0.05$, and from $24 \pm 9$ to $21 \pm 11 \mathrm{~ms}, \mathrm{p}=\mathrm{NS}$, in progressors and non-progressors, respectively). Thus, to further explore the associa- 
Table 3. Predictors of CAC progression at multivariable analysis

\begin{tabular}{llllll}
\hline Variable & Unit increment & $\beta$-Coefficient & SE & OR (95\% CI) & p \\
\hline Age & per year increment & 0.23 & 2.76 & $0.64(0.45-1.73)$ & NS \\
Hypertension & presence vs. absence & 0.65 & 2.23 & $0.91(0.75-1.24)$ & NS \\
Diabetes & presence vs. absence & 1.22 & 0.89 & $1.17(1.13-1.28)$ & 0.01 \\
ASCVD & presence vs. absence & 0.93 & 1.61 & $1.05(0.91-1.29)$ & NS \\
CRP & 5-mg/dl increase & 1.14 & 0.98 & $1.09(1.04-1.18)$ & 0.05 \\
LDL cholesterol & 10-mg/dl increase & 1.09 & 0.77 & $1.07(1.01-1.12)$ & 0.05 \\
Sevelamer use & presence vs. absence & 0.071 & 0.65 & $0.66(0.36-0.81)$ & 0.001 \\
Baseline CAC & 10-unit increment & 1.11 & 0.95 & $1.08(1.05-1.19)$ & 0.05 \\
\hline
\end{tabular}

NS = Not significant. For further abbreviations, see table 1.

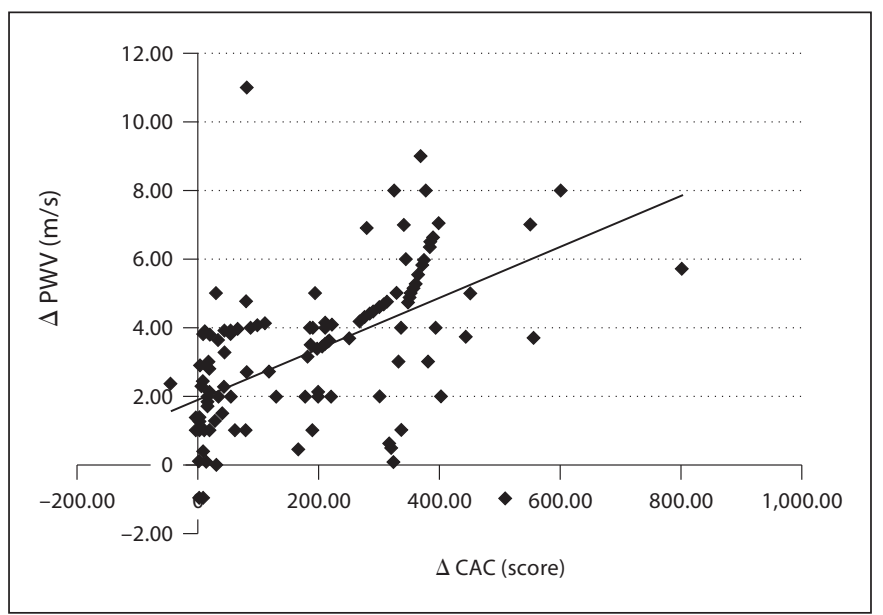

Fig. 2. Correlation between $C A C$ progression $(\triangle \mathrm{CAC})$ and $\mathrm{PWV}$ $(\Delta \mathrm{PWV})$ changes during follow-up (CAC is expressed in Agatston score unit and $\mathrm{PWV}$ is expressed in $\mathrm{m} / \mathrm{s}$ ).

tion between CAC and other markers of CV disease, we plotted the relative change of CAC with changes in PWV and QTd, as shown in figures 2 and 3, respectively. Indeed, there was a significant moderate linear correlation between CAC and PWV $(r=0.482, \mathrm{p}<0.0001)$ and QTd $(\mathrm{r}=0.419, \mathrm{p}<0.001)$ progression.

Multivariable analyses confirmed the independent associations of CAC progression with changes in PWV and QTd (tables 4, 5). Specifically, after adjustment for confounders, every 20 -unit increase of CAC corresponded to a significant $23 \%(95 \%$ CI 1.12-1.27; p < 0.001) and 32\% (95\% CI 1.09-1.37; $\mathrm{p}<0.01)$ increase in the risk of experiencing a $1-\mathrm{m} / \mathrm{s}$ increase in PWV and $1 \mathrm{~ms}$ in QTd, respectively. Notably, these results were unchanged when QTc or QTd were modeled (data not shown).

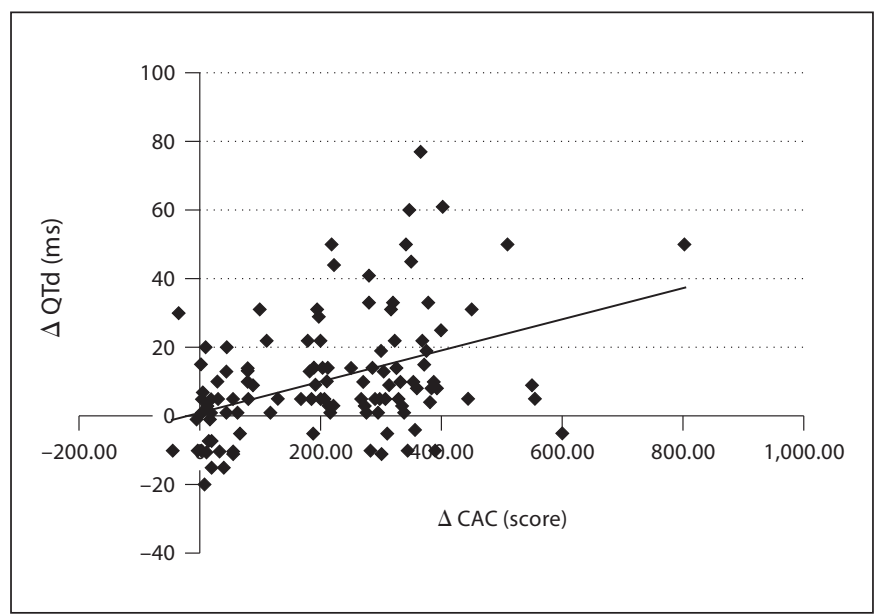

Fig. 3. Correlation between CAC progression ( $\triangle$ CAC) and QTd changes $(\Delta \mathrm{QTd})$ during follow-up (CAC is expressed in Agatston score unit and QTd is expressed in $\mathrm{ms}$ ).

\section{Discussion}

The most notable finding of these results is that CAC progression is associated with a progressive deterioration of parameters of arterial stiffness and cardiac repolarization. Previous cross-sectional studies have already documented that VCs are closely associated with arterial stiffness $[10,19]$ and in a previous paper we found an association between CAC and QTd [14]. However, these analyses add to the existing body of evidence by confirming these associations in a longitudinal study.

It is well recognized that $\mathrm{VC}$ represents a stigmata for patients undergoing CKD-5 [20-23]. Data show that about 60 and $80 \%$ of incident and prevalent patients on chronic dialysis have evidence of CAC [21-23]. CAC is a 
Table 4. Predictors of a 1-m/s increase in PWV progression in multivariable analysis

\begin{tabular}{lllllc}
\hline Variable & Unit increment & $\beta$-Coefficient & SE & OR (95\% CI) & $\mathrm{p}$ \\
\hline Age & per year increment & 0.56 & 3.3 & $1.13(0.89-1.44)$ & NS \\
Hypertension & presence vs. absence & 1.1 & 2.4 & $1.03(0.76-1.23)$ & NS \\
Diabetes & presence vs. absence & 0.23 & 2.76 & $0.64(0.45-1.73)$ & NS \\
ASCVD & presence vs. absence & 0.93 & 1.61 & $1.05(0.91-1.29)$ & NS \\
CRP & 5-mg/dl increase & 1.11 & 0.56 & $1.09(1.02-1.12)$ & $<0.05$ \\
LDL cholesterol & 10-mg/dl increase & 1.33 & 0.67 & $1.19(1.13-1.33)$ & $<0.05$ \\
CAC progression & 20-unit increase & 1.32 & 0.74 & $1.23(1.12-1.27)$ & $<0.001$ \\
Sevelamer use & presence vs. absence & 0.71 & 0.61 & $0.86(0.83-0.91)$ & 0.05 \\
\hline
\end{tabular}

NS = Not significant. For further abbreviations, see table 1.

Table 5. Predictors of a 1-ms increase in QTd in multivariable analyses

\begin{tabular}{llllll}
\hline Variable & Unit increment & $\beta$-Coefficient & SE & OR (95\% CI) & $\mathrm{p}$ \\
\hline Age & per year increment & 0.77 & 0.19 & $0.84(0.76-0.97)$ & NS \\
Hypertension & presence vs. absence & 0.82 & 1.39 & $1.04(0.92-1.33)$ & NS \\
Diabetes & presence vs. absence & 1.02 & 5.43 & $1.09(0.89-1.34)$ & NS \\
ASCVD & presence vs. absence & 0.71 & 1.41 & $1.14(1.04-1.18)$ & NS \\
CAC progression & 20-unit increase & 1.37 & 0.61 & $1.32(1.09-1.37)$ & $<0.01$ \\
CRP & 5-mg/dl increase & 1.21 & 0.71 & $1.14(1.04-1.18)$ & $<0.05$ \\
LDL cholesterol & 10-mg/dl increase & 1.15 & 0.87 & $1.24(1.11-1.29)$ & $<0.05$ \\
Sevelamer use & presence vs. absence & 0.058 & 0.013 & $0.64(0.45-0.74)$ & $<0.001$ \\
\hline
\end{tabular}

NS = Not significant. For further abbreviations, see table 1.

marker of vasculopathy and portends poor prognosis in the general population as well as in high-risk populations. Matsuoka et al. [12] documented a linear association between the CAC burden and the risk of death in a cohort of 104 chronic hemodialysis patients (adjusted relative death risk 1.001; 95\% CI 1.000-1.002; $\mathrm{p}=0.0003$, per unit increase of CAC) [12]. Similarly, Block et al. [11] concluded that baseline CAC score was a significant and powerful predictor of mortality in a cohort of 127 patients new to dialysis after adjustment for age, race, gender, and diabetes (hazard ratio for CAC $>400$ vs. CAC $=0: 4.5 ; 95 \%$ CI 1.33-15.14; $\mathrm{p}=0.016$ ) [11]. However, CAC does not appear to be an accurate marker of coronary artery stenosis and the debate on how VC might predispose to CV mortality is still an open one [24].

Previous publications have indicated that the severity of CAC and calcification of the aorta are strictly connected to PWV in both predialysis and dialysis patients [10, $25,26]$. Decreased arterial compliance is the result of many contributing factors such as change in the collagen- elastin ratio, atherosclerotic plaques as well as calcification of the intima or the media layer of the vessel wall. Current results add to the existing body of evidence, by confirming that those patients who experience an increase in CAC are also likely to experience a significant deterioration of the arterial compliance of large conduit arteries. Of note, an increase in PWV has been associated with an excess of morbidity and mortality in the general population and in CKD-5 patients $[15,16]$. Blacher et al. [15] documented that aortic stiffness determined by measurement of aortic PWV was linearly associated with allcause and CV mortality in a cohort of 241 CKD-5 patients (adjusted OR per 1-m/s increase in all-cause mortality 1.39; 95\% CI 1.19-1.62). Thus, CAC should be regarded as a useful aid in providing a global assessment of the CV risk rather than a surrogate for coronary stenosis $[11,12]$.

A few peculiarities of this study deserve mention. Contrary to what was previously documented by Block et al. [11], we observed that even zero scores at dialysis entry 
might progress over time. Nonetheless, in keeping with previous evidence, baseline CAC burden proved to be an independent predictor of progression. In fact, the greater the Agatston CAC score at baseline, the higher the likelihood of experiencing a true progression by study completion. This finding may suggest that a patient entering dialysis with no evidence of CAC accrues calcium at a lower speed than a peer with a greater burden of calcification rather than being protected from CAC deposition and progression. In other words, zero scores are less susceptible to vascular deterioration rather than being protected from VCs.

As documented by the United States Renal Data System, congestive heart failure and sudden death are the most frequent causes of $\mathrm{CV}$ death in CKD-5, followed by acute myocardial infarction, stroke, and peripheral arterial disease (http://www.usrds.org/2006/ref/H_morte_ 06.pdf). Different factors have been investigated to explain the pathogenesis of cardiac arrhythmias that lead to sudden cardiac death in hemodialysis [27]. In particular, coronary artery disease, arterial hypertension, congestive heart failure, autonomic neuropathy and uremic cardiomyopathies might induce a significant cardiac remodeling that affects the number and functionality of cardiac ion channels [27]. Specifically, it has been postulated that patients undergoing hemodialysis might experience a progressive diminution and inhibition of the potassium channels that might lead to a reduction of the 'cardiac repolarization reserve' [27]. Several drugs and the acute changes in levels of serum calcium and potassium that occur during a dialysis section further exacerbate the risk of cardiac arrhythmias as documented by changes in the QT interval on ECG (http://www.qtdrugs.org). It has been suggested that QTd reflects heterogeneity of myocardial repolarization and predicts poor all-cause and CV survival independently on traditional CV risk factors in CKD-5 [27-33]. In a previous paper we showed an independent association between CAC and QTd, suggesting that subclinical vasculopathy might also be related to cardiac electrical impairments [14]. In this perspective, a couple of studies from the general population deserve mention. Scherer et al. [34] showed, in a large cohort of 848 men and 156 women, a significant association between CAC and ECG abnormalities independent of traditional CV risk factors [34]. In another paper, Edvardsen et al. [35] concluded that CAC is significantly associated with regional left ventricular dysfunction in the corresponding perfusion territory among individuals with no history of heart disease. Notably the current results further corroborate these findings and suggest that patients experiencing progression of the VC burden in the coronaries are at high risk of developing cardiac repolarization abnormalities and, possibly, arrhythmias when compared to peers with stable CAC.

Taken together, these results suggest that coronary calcifications are associated with stiffness of large central arteries and cardiac repolarization defects. Thus, we might speculate that VC predisposes to tissue ischemia and cardiac arrhythmia independently of the presence of an arterial stenosis. Indeed, aortic stiffness that results from VC [19], predisposes to left ventricular remodeling $[26,36]$ and low diastolic arterial pressure leading to increase oxygen demand, reduced coronary perfusion and cardiac repolarization abnormalities.

A few limitations have to be taken into account when these results are interpreted. The relatively small sample size and the low reproducibility of the mean QTd limit the generalizability of these findings. Furthermore, although prospective, this is not a randomized trial and potential confounders might have influenced our results. We could not account for the presence of left ventricular hypertrophy that was not assessed in our study cohort. Nonetheless, considering that this is a prospective study and every patient is at the same time case and control, it is unlikely that left ventricular hypertrophy may have biased our results. The high homogeneity of the study cohort and the number of markers of CV disease are, in the authors' opinion, the major strengths of this study. Finally, due to the random selection of this series of patients from the INDEPENDENT study cohort and the lack of clinical difference between this subset of patients and the rest of the INDEPENDENT study cohort (data not shown), it is unlikely that increasing the sample size would affect current results.

In conclusion, this study provides evidence of the close association of functional and morphological vascular disease markers. Patients experiencing CAC progression were at significantly greater risk of experiencing a simultaneous deterioration of markers of arterial compliance and cardiac repolarization. These results indicate that CAC could represent a step in the continuum of events responsible for the CV mortality in CKD-5. Nonetheless, future studies should confirm that preventing or attenuating CAC progression results in a better prognosis among people receiving dialysis. 


\section{References}

-1 Foley RN, Parfrey PS, Sarnak MJ: Epidemiology of cardiovascular disease in chronic renal disease. J Am Soc Nephrol 1998;9:S16S23.

-2 Block GA, Klassen PS, Lazarus JM, Ofsthun N, Lowrie EG, Chertow GM: Mineral metabolism, mortality, and morbidity in maintenance hemodialysis. J Am Soc Nephrol 2004; 15:2208-2218.

3 Kalantar-Zadeh K, Kuwae N, Regidor DL, Kovesdy CP, Kilpatrick RD, Shinaberger CS, McAllister CJ, Budoff MJ, Salusky IB, Kopple JD: Survival predictability of time-varying indicators of bone disease in maintenance hemodialysis patients. Kidney Int 2006;70: 771-780.

$\checkmark 4$ Covic A, Kothawala P, Bernal M, Robbins S, Chalian A, Goldsmith D: Systematic review of the evidence underlying the association between mineral metabolism disturbances and risk of all-cause mortality, cardiovascular mortality and cardiovascular events in chronic kidney disease. Nephrol Dial Transplant 2009;24:1506-1523.

$\checkmark 5$ Moe S, Drueke T, Cunningham J, Goodman W, Martin K, Olgaard K, Ott S, Sprague S, Lameire N, Eknoyan G: Definition, evaluation, and classification of renal osteodystrophy: a position statement from kidney disease: improving global outcomes (KDIGO). Kidney Int 2006;69:1945-1953.

-6 Jono S, McKee MD, Murry CE, Shioi A, Nishizawa Y, Mori K, Morii H, Giachelli CM: Phosphate regulation of vascular smooth muscle cell calcification. Circ Res 2000; 87:E10-E17.

7 Giachelli CM: Vascular calcification: in vitro evidence for the role of inorganic phosphate. J Am Soc Nephrol 2003;14:S300-S304.

$\checkmark 8$ Li X, Yang HY, Giachelli CM: Role of the sodium-dependent phosphate cotransporter, Pit-1, in vascular smooth muscle cell calcification. Circ Res 2006;14:905-912.

-9 Yang H, Curinga G, Giachelli CM: Elevated extracellular calcium levels induce smooth muscle cell matrix mineralization in vitro. Kidney Int 2004;66:2293-2299.

-10 Guerin AP, London GM, Marchais SJ, Metivier F: Arterial stiffening and vascular calcifications in end-stage renal disease. Nephrol Dial Transplant 2000;15:1014-1021.

- 11 Block GA, Raggi P, Bellasi A, Kooienga L, Spiegel DM: Mortality effect of coronary calcification and phosphate binder choice in incident hemodialysis patients. Kidney Int 2007;71:438-441.

-12 Matsuoka M, Iseki K, Tamashiro M, Fujimoto N, Higa N, Touma T, Takishita S: Impact of high coronary artery calcification score on survival in patients on chronic hemodialysis. Clin Exp Nephrol 2004;8:54-58.
13 Detrano R, Guerci AD, Carr JJ, Bild DE, Burke G, Folsom AR, Liu K, Shea S, Szklo M, Bluemke DA, O'Leary DH, Tracy R, Watson K, Wong ND, Kronmal RA: Coronary calcium as a predictor of coronary events in four racial or ethnic groups. N Engl J Med 2008; 358:1336-1345.

14 Di Iorio BR, Bortone S, Piscopo C, Grimaldi P, Cucciniello E, D’Avanzo E, Mondillo F, Cillo N, Bellizzi V: Cardiac vascular calcification and QT interval in ESRD patients: is there a link? Blood Purif 2006;24:451-459.

15 Blacher J, Guerin AP, Pannier B, Marchais SJ, Safar ME, London GM: Impact of aortic stiffness on survival in end-stage renal disease. Circulation 1999;99:2434-2439.

16 Willum Hansen T, Staessen JA, Torp-Pedersen C, Rasmussen S, Thijs L, Ibsen H, Jeppesen J: Prognostic value of aortic pulse wave velocity as index of arterial stiffness in the general population. Circulation 2006;113: 664-670.

17 Di Iorio BR, Cucciniello E, Bellizzi V: Vascular calcification and QT interval in incident hemodialysis patients. J Nephrol 2009;22: 694-698.

18 Bellasi A, Kooienga L, Block GA, Veledar E, Spiegel DM, Raggi P: How long is the warranty period for nil or low coronary artery calcium in patients new to hemodialysis? J Nephrol 2009;22:255-262.

19 Raggi P, Bellasi A, Ferramosca E, Islam T, Muntner P, Block GA: Association of pulse wave velocity with vascular and valvular calcification in hemodialysis patients. Kidney Int 2007;71:802-807.

20 Goldsmith D, Ritz E, Covic A: Vascular calcification: a stiff challenge for the nephrologist. Does preventing bone disease cause arterial disease? Kidney Int 2004;66:1315-1333.

21 Block GA, Spiegel DM, Ehrlich J, Mehta R, Lindbergh J, Dreisbach A, Raggi P: Effects of sevelamer and calcium on coronary artery calcification in patients new to hemodialysis. Kidney Int 2005;68:1815-1824.

22 Chertow GM, Burke SK, Raggi P: Sevelamer attenuates the progression of coronary and aortic calcification in hemodialysis patients. Kidney Int 2002;62:245-252.

23 Spiegel DM, Raggi P, Mehta R, Lindberg JS, Chonchol M, Ehrlich J, James G, Chertow GM, Block GA: Coronary and aortic calcifications in patients new to dialysis. Hemodial Int 2004;8:265-272.

24 Haydar AA, Covic A, Colhoun H, Rubens M, Goldsmith DJ: Coronary artery calcification and aortic pulse wave velocity in chronic kidney disease patients. Kidney Int 2004;65: 1790-1794.
25 Raggi P, Bellasi A, Ferramosca E, Block GA, Muntner P: Pulse wave velocity is inversely related to vertebral bone density in hemodialysis patients. Hypertension 2007;49:12781284.

26 Lemos MM, Jancikic AD, Sanches FM, Christofalo DM, Ajzen SA, Miname MH, Santos RD, Fachini FC, Carvalho AB, Draibe SA, Canziani ME: Pulse wave velocity - a useful tool for cardiovascular surveillance in pre-dialysis patients. Nephrol Dial Transplant 2007;22:3527-3532.

27 Gussak I, Gussak HM: Sudden cardiac death in nephrology: focus on acquired long QT syndrome. Nephrol Dial Transplant 2007;22: $12-14$.

28 Wu VC, Lin LY, Wu KD: QT interval dispersion in dialysis patients. Nephrology (Carlton) $2005 ; 10: 109-112$.

29 Bleyer AJ, Hartman J, Brannon PC, ReevesDaniel A, Satko SG, Russell G: Characteristics of sudden death in hemodialysis patients. Kidney Int 2006;69:2268-2273.

$>30$ Karnik JA, Young BS, Lew NL, Herget M, Dubinsky C, Lazarus JM, Chertow GM: Cardiac arrest and sudden death in dialysis units. Kidney Int 2001;60:350-357.

31 Bleyer AJ, Russell GB, Satko SG: Sudden and cardiac death rates in hemodialysis patients. Kidney Int 1999;55:1553-1559.

>32 Covic A, Diaconita M, Gusbeth-Tatomir P, Covic M, Botezan A, Ungureanu G, Goldsmith DJ: Haemodialysis increases QTc interval but not QTc dispersion in ESRD patients without manifest cardiac disease. Nephrol Dial Transplant 2002;17:2170-2177.

33 Kanbay M, Afsar B, Goldsmith D, Covic A: Sudden death in hemodialysis: an update. Blood Purif 2010;30:135-145.

34 Scherer ML, Aspelund T, Sigurdsson S, Detrano R, Garcia M, Mitchell GF, Launer LJ, Thorgeirsson G, Gudnason V, Harris TB: Abnormal T-wave axis is associated with coronary artery calcification in older adults. Scand Cardiovasc J 2009;43:240-248.

-35 Edvardsen T, Detrano R, Rosen BD, Carr JJ, Liu K, Lai S, Shea S, Pan L, Bluemke DA, Lima JA: Coronary artery atherosclerosis is related to reduced regional left ventricular function in individuals without history of clinical cardiovascular disease: the multiethnic study of atherosclerosis. Arterioscler Thromb Vasc Biol 2006;26:206-211.

-36 Nitta K, Akiba T, Uchida K, Otsubo S, Otsubo Y, Takei T, Ogawa T, Yumura W, Kabaya $\mathrm{T}$, Nihei $\mathrm{H}$ : Left ventricular hypertrophy is associated with arterial stiffness and vascular calcification in hemodialysis patients. Hypertens Res 2004;27:47-52. 